AEI-2009-062

ULB-TH/09-23

\title{
Supersymmetric quantum cosmological billiards
}

\author{
Axel Kleinschmidt \\ Physique Théorique et Mathématique 83 International Solvay Institutes, \\ Université Libre de Bruxelles, Boulevard du Triomphe, ULB-CP231, BE-1050 Bruxelles, Belgium
}

Michael Koehn and Hermann Nicolai

Max-Planck-Institut für Gravitationsphysik, Albert-Einstein-Institut, Am Mühlenberg 1, DE-14476 Golm, Germany

\begin{abstract}
$D=11$ Supergravity near a space-like singularity admits a cosmological billiard description based on the hyperbolic Kac-Moody group $E_{10}$. The quantization of this system via the supersymmetry constraint is shown to lead to wavefunctions involving automorphic (Maass wave) forms under the modular group $W^{+}\left(E_{10}\right) \cong P S L_{2}(0)$ with Dirichlet boundary conditions on the billiard domain. A general inequality for the Laplace eigenvalues of these automorphic forms implies that the wave function of the universe is generically complex and always tends to zero when approaching the initial singularity. We discuss possible implications of this result for the question of singularity resolution in quantum cosmology and comment on the differences with other approaches.
\end{abstract}

PACS numbers: 04.60.-m, 04.60.Kz, 04.65.+e, 98.80.Qc

One of the surprising results in the study of classical gravity very close to a generic space-like singularity due to Belinskii, Khalatnikov and Lifshitz (BKL) 1] was the realization that, under rather general assumptions, the system becomes ultralocal in space and can be described by a sequence of Kasner regimes. In the strict limit towards the singularity, the Kasner behavior is interspersed with hard reflections of the logarithms of the spatial scale factors off infinite potential walls [2, 3]. This behavior has been termed 'cosmological billiards'; the geometry of the billiard table as well as the possible occurrence of chaotic oscillations near the singularity depend on the dimension and the matter content of the theory [4, 5]. In particular, for $D=11$ supergravity it was shown by Damour and Henneaux [6] that the billiard domain is the fundamental Weyl chamber $\mathcal{C}$ of the hyperbolic Kac-Moody group $E_{10}$, implying chaotic behavior.

Resolving the cosmological singularity requires the transition to the quantum theory. In this paper we address this issue in the framework of cosmological billiards. More precisely, we will set up and solve the quantum constraints for $D=11$ supergravity for the ten spatial scale factors and the fermionic degrees of freedom in compliance with the supersymmetry constraint. The approach followed here is thus a variant of 'minisuperspace' quantization of gravity pioneered in $[7,8,9,9$ and further developed in 10, 11, 12, 13, 14, 15. An essential new ingredient of the present work is the arithmetic structure provided by $E_{10}$ and its Weyl group, whose relevance in the context of Einstein gravity was pointed out and explored in [16]. In accordance with the M-theory proposal of [17], where a correspondence was established at the classical level between a (truncated) gradient expansion of the $D=11$ supergravity equations of motion and an expansion in heights of roots of a constrained 'geodesic' $E_{10} / K\left(E_{10}\right)$ coset space model, the cosmological billiards approximation corresponds to the restriction of the coset model to the Cartan subalgebra of $E_{10}$. Our results therefore represent the first step towards the quantization of the full coset model.

Starting with the bosonic variables, the diagonal metric considered for the cosmological billiard for a $(d+1)$ dimensional space-time is of the form [18] (for $d \geq 3$ )

$$
d s^{2}=-N^{2} d t^{2}+\sum_{a=1}^{d} e^{-2 \beta^{a}} d x_{a}^{2},
$$

leading to the kinetic term $\mathcal{L}_{\text {kin }}=\frac{1}{2} n^{-1} \sum_{a, b=1}^{d} \dot{\beta}^{a} G_{a b} \dot{\beta}^{b}$ in terms of the lapse $n=N g^{-1}$ (the spatial volume is $\left.g^{1 / 2}=\exp \left[-\sum_{a} \beta^{a}\right]\right)$ and the Lorentzian DeWitt metric 19]

$$
\dot{\beta}^{a} G_{a b} \dot{\beta}^{b} \equiv \sum_{a=1}^{d}\left(\dot{\beta}^{a}\right)^{2}-\left(\sum_{a=1}^{d} \dot{\beta}^{a}\right)^{2} .
$$

It will be essential that for $d=10$ this metric coincides with the restriction of the Cartan-Killing metric of $E_{10}$ to its Cartan subalgebra. The spatial ultralocality of the BKL limit reduces the gravitational model to a classical mechanics system of a relativistic billiard ball described by the $\beta^{a}$ variables moving on straight null lines in the Lorentzian space with metric $G_{a b}$ until hitting a billiard table wall. The straight line segments are Kasner regimes. The singularity is at $t=+\infty$ in the 'Zeno-like' time co-ordinate $t$ that is related to physical (proper) time $T$ by $t \sim-\log T$. There is one such system for each spatial point $\mathbf{x}$, and these systems are all decoupled.

The conjugate canonical bosonic variables of the billiard system are $\beta^{a}$ and $\pi_{a}=G_{a b} \dot{\beta}^{b}$; the Hamiltonian then is $\mathcal{H}=\frac{1}{2} \pi_{a} G^{a b} \pi_{b}$ with the inverse metric $G^{a b}$, and we have set the lapse $n=1$. Before quantisation, we perform the following change of variables by means of which the billiard motion can be projected onto the unit hyperboloid in $\beta$-space [4]

$$
\beta^{a}=\rho \omega^{a}, \quad \omega^{a} G_{a b} \omega^{b}=-1, \quad \rho^{2}=-\beta^{a} G_{a b} \beta^{b},
$$

where $\rho$ is the 'radial' direction in the future light-cone and $\omega^{a}=\omega^{a}(z)$ are expressible as functions of $d-1$ 
coordinates $z$ on the unit hyperboloid. The limit towards the singularity is $\rho \rightarrow \infty$ in these variables. The WheelerDeWitt (WDW) operator on $\beta$-space takes the form [20]

$$
\mathcal{H} \equiv G^{a b} \partial_{a} \partial_{b}=-\rho^{1-d} \frac{\partial}{\partial \rho}\left(\rho^{d-1} \frac{\partial}{\partial \rho}\right)+\rho^{-2} \Delta_{\mathrm{LB}},
$$

where $\Delta_{\mathrm{LB}}$ is the Laplace-Beltrami operator on the $(d-1)$-dimensional unit hyperboloid. The WDW equation therefore reads $\mathcal{H} \Phi(\rho, z)=0$ for the wavefunction $\Phi(\rho, z)$. As usual (see e.g. [15]) one can adopt $\rho$ as a time coordinate in the initially 'timeless' WDW equation, with the standard (Klein-Gordon-like) invariant inner product

$$
\left(\Phi_{1}, \Phi_{2}\right)=i \int d \Sigma^{a} \Phi_{1}^{*} \stackrel{\leftrightarrow}{\partial_{a}} \Phi_{2}
$$

where the integral is to be taken over a spacelike hypersurface inside the forward lightcone in $\beta$-space.

In order to construct solutions we separate variables by means of the ansatz $\Phi(\rho, z)=R(\rho) F(z)[9$, 11]. For any eigenfunction $F(z)$ obeying

$$
-\Delta_{\mathrm{LB}} F(z)=E F(z)
$$

the associated radial equation is solved by

$$
R_{ \pm}(\rho)=\rho^{-\frac{d-2}{2}} e^{ \pm i \sqrt{E-\left(\frac{d-2}{2}\right)^{2}} \log \rho} .
$$

Positive frequency waves emanating from the singularity correspond to $R_{-}(\rho)$ and have positive inner product (5). To study the eigenvalues of the Laplace-Beltrami operator on the unit hyperboloid we use a generalized upper half plane model $z=(\vec{u}, v)$ for the unit hyperboloid with co-ordinates $\vec{u} \in \mathbb{R}^{d-2}$ and $v \in \mathbb{R}_{>0}$ and the Poincaré metric $d s^{2}=v^{-2}\left(d v^{2}+d \vec{u}^{2}\right)$ such that

$$
\Delta_{\mathrm{LB}}=v^{d-1} \partial_{v}\left(v^{3-d} \partial_{v}\right)+v^{2} \partial_{\vec{u}}^{2} .
$$

For the spectral problem we must specify boundary conditions. For the cosmological billiard, these are provided by infinite ('sharp') potential walls which encapsulate the effect of spatial inhomogeneities and matter fields near the spacelike singularity, as explained in [4, 5]. Following the original suggestion of [9], we are thus led to impose the vanishing of the wavefunction on the boundary of the fundamental domain specified by these walls. Accordingly, let $F(z)$ be any function on the hyperboloid satisfying (6) with Dirichlet conditions at the boundaries of this domain [21]. A direct generalization of the arguments on page 28 of Ref. [22] gives

$$
-\left(\Delta_{\mathrm{LB}} F, F\right) \geq \int d v d^{d-2} u v^{3-d}\left(\partial_{v} F\right)^{2}
$$

with (6) and (8). Considering also

$$
\begin{aligned}
(F, F) & =\int d v d^{d-2} u v^{1-d} F^{2} \\
& =\frac{2}{d-2} \int d v d^{d-2} u v^{2-d} F \partial_{v} F,
\end{aligned}
$$

the Cauchy-Schwarz inequality entails

$$
E \geq\left(\frac{d-2}{2}\right)^{2} \text {. }
$$

From the explicit solution (7) we thus conclude that $R_{ \pm}(\rho) \rightarrow 0$ when $\rho \rightarrow \infty$, and therefore the full wavefunction and all its $\rho$ derivatives tend to zero near the singularity. While this conclusion also holds with Neumann boundary conditions (for which $E \geq 0$ ), the above inequality furthermore ensures that the full wavefunction is generically complex and oscillating.

Let us now return to maximal supergravity, where the wavefunctions can be further analyzed by exploiting the underlying symmetry encoded in the Weyl group $W\left(E_{10}\right)$ and its arithmetic properties, and in particular the new links between hyperbolic Weyl groups and generalized modular groups uncovered in 23]. The Weyl reflections that the classical particle is subjected to when colliding with one of the walls are norm preserving, and therefore the reflections can be projected to any hyperboloid of constant $\rho$, inducing a non-linear action on the coordinates $z$ (given in (13) below for the fundamental reflections). For physical amplitudes to be invariant under the Weyl group, the full wavefunction (in $\beta$-space) must transform as follows

$$
\Phi(\rho, z)= \pm \Phi\left(\rho, w_{I} \cdot z\right)
$$

for the ten generating fundamental reflections $w_{I}$ of $W\left(E_{10}\right)$, labeled by $I=-1,0,1, \ldots, 8$. Restricting the wavefunction to the fundamental Weyl chamber, one easily checks that the plus sign in (12) corresponds to Neumann boundary conditions, and the minus sign to Dirichlet conditions (which we adopt here). $\Phi(\rho, z)$ is thus invariant under even Weyl transformations $s \in W^{+}\left(E_{10}\right)$ irrespective of the chosen boundary conditions.

Choosing coordinates as in (8) the relevant variables now live in a 9-dimensional 'octonionic upper half plane' with $z=u+\mathrm{i} v$ where $u \equiv \vec{u} \in \mathbb{O}$ is an octonion. The ten fundamental reflections of $W\left(E_{10}\right)$ act as $(j=1, \ldots, 8)$

$$
w_{-1}(z)=\frac{1}{\bar{z}}, w_{0}(z)=-\theta \bar{z} \theta+\theta, w_{j}(z)=-\varepsilon_{j} \bar{z} \varepsilon_{j}
$$

where $\bar{z}:=\bar{u}-\mathrm{i} v$, with $\mathrm{i} u=\bar{u} \mathrm{i}$ in accordance with Cayley-Dickson doubling 24]. $\varepsilon_{j}$ and $\theta$, respectively, are the simple roots and the highest root of $E_{8}$ expressed as unit octonions [23, 24]. We note that for $u \in \mathbb{R}$ the formulas (13) reduce to the ones familiar from complex analysis, with $z \mapsto 1 / \bar{z}, z \mapsto-\bar{z}+1$ and $z \mapsto-\bar{z}$, generating the group $P G L_{2}(\mathbb{Z})$. For even Weyl transformations, we re-obtain the standard modular group $P S L_{2}(\mathbb{Z})$ generated by $S(z)=\left(w_{-1} w_{1}\right)(z)=-1 / z$ and $T(z)=\left(w_{0} w_{1}\right)(z)=z+1$. Similarly, the even Weyl group $W^{+}\left(E_{10}\right)$ is isomorphic to $P S L_{2}(0)$ where 0 are the integer octonions ('octavians', see [24]) and the group $P S L_{2}(\mathrm{O})$ is defined by iterating the action of (13) an even number of times [23]. Consequently, for maximal supergravity the bosonic wavefunctions $\Phi(\rho, z)$ are odd 
Maass wave forms for $P S L_{2}(0)$, that is, invariant eigenfunctions of the Laplace-Beltrami operator transforming with a minus sign in (12) under the extension $W\left(E_{10}\right)$ of $P S L_{2}(0)$. Understanding these modular functions remains an outstanding mathematical challenge, see 25] for an introduction (and [22] for the $P S L_{2}(\mathbb{Z})$ theory). For the groups $P S L_{2}(\mathbb{Z})$ and $P S L_{2}(\mathbb{Z}[i])$ the (purely discrete) spectra of odd Maass wave forms have been investigated numerically in [26, 27, 28, 29, 30]. By modular invariance, the wavefunctions can be restricted to the fundamental domain of the action of $W\left(E_{10}\right)$, and conversely, their modular property defines them on the whole hyperboloid. The Klein-Gordon inner product (5) must likewise be restricted to the fundamental chamber

$$
\left(\Phi_{1}, \Phi_{2}\right)=i \int_{\mathcal{F}} d \operatorname{vol}(z) \rho^{d-1} \Phi_{1}^{*} \stackrel{\leftrightarrow}{\partial_{\rho}} \Phi_{2}
$$

where $\mathcal{F}$ is the intersection of $\mathcal{C}$ with the unit hyperboloid. This is the canonical quantum gravity analog of the one-loop amplitude in string theory which is rendered finite upon 'division' by the modular group $P S L_{2}(\mathbb{Z})$.

We now turn to the extension of the quantum billiard analysis to maximal supergravity and restrict to $d=10$ henceforth [31, 32, 33]. Classically, the gravitino $\psi_{\mu}$ of $D=11$ supergravity performs a separate fermionic billiard motion [34]. This is most easily expressed in a supersymmetry gauge $\psi_{t}=\Gamma_{t} \Gamma^{a} \psi_{a}$ 31] and in the variables [34] (with $\left.\Gamma_{*}=\Gamma^{1} \cdots \Gamma^{10}\right)$

$$
\varphi^{a}=g^{1 / 4} \Gamma_{*} \Gamma^{a} \psi^{a} \quad(\text { no sum on } a=1, \ldots, 10) .
$$

Using (15) the Dirac brackets between two gravitino variables (see (6.3) in [33]) become $\left\{\varphi_{\alpha}^{a}, \varphi_{\beta}^{b}\right\}=-2 i G^{a b} \delta_{\alpha \beta}$, where we have written out the 32 real spinor components using the indices $\alpha, \beta$. The fermionic and bosonic variables are linked by the supersymmetry constraint

$$
\mathcal{S}_{\alpha} \equiv \sum_{a, b=1}^{10} \dot{\beta}^{a} G_{a b} \varphi_{\alpha}^{b}=\sum_{a=1}^{10} \pi_{a} \varphi_{\alpha}^{a}=0 .
$$

The supersymmetry constraint implies the Hamiltonian constraint $\mathcal{H}=0$ by closure of the algebra

$$
\frac{1}{4}\left\{\mathcal{S}_{\alpha}, \mathcal{S}_{\beta}\right\}=\delta_{\alpha \beta} \mathcal{H}
$$

In order to quantize this system we rewrite the 320 real gravitino components $\varphi_{\alpha}^{a}$ in terms of 160 complex ones, and replace the Dirac brackets by canonical anticommutators to obtain a fermionic Fock space of dimension $2^{160}$ over the vacuum $|\Omega\rangle$. For the supersymmetry constraint this amounts to the redefinition $\tilde{\mathcal{S}}_{A}=\mathcal{S}_{A}+i \mathcal{S}_{A+16}$ for $A=1, \ldots, 16$. The quantum constraint is then solved by

$$
|\Psi\rangle=\prod_{A=1}^{16} \tilde{\mathcal{S}}_{A}^{\dagger}(\Phi(\rho, z)|\Omega\rangle),
$$

(with $\tilde{\mathcal{S}}_{A}|\Omega\rangle=0$ ) if and only if the function $\Phi(\rho, z)$ is a solution of the WDW equation $\mathcal{H} \Phi=0$. While this solution is close to the 'bottom of the Dirac sea', there is an analogous one 'close to the top' with $\tilde{\mathcal{S}}_{A}^{\dagger}$ replaced by $\tilde{\mathcal{S}}_{A}$ and $|\Omega\rangle$ by the completely filled state.

The cosmological billiards description is very useful but takes into account the dependence on spatial inhomogeneities and matter degrees of freedom only in a very rudimentary way via the infinite potential walls. It would thus be desirable to develop an approximation scheme for the quantum state in line with the 'small tension' expansion proposed in [17], and thereby hopefully resolve the difficulties encountered in extending the 'dictionary' of 17] to higher order spatial gradients and heights of roots in a quantum mechanical context. In the BKL approximation, the full wavefunction is expected to factorize as

$$
\left|\Psi_{\text {full }}\right\rangle \sim \prod_{\mathbf{x}}\left|\Psi_{\mathbf{x}}\right\rangle
$$

near the singularity into a formal product over wavefunctions of the type (18), one for each spatial point (with independent bosonic wavefunctions $\Phi_{\mathbf{x}}(\rho(\mathbf{x}), z(\mathbf{x})$ ) and space-dependent diagonal metric variables $\beta^{a}(\mathbf{x}) \equiv$ $(\rho(\mathbf{x}), z(\mathbf{x}))$. The task is then to replace the formal expression (19) by a wavefunction depending on the (infinite) tower of $E_{10}$ degrees of freedom, effectively implementing the de-emergence of space and time near the cosmological singularity, and their replacement by purely algebraic concepts [31, 35]. We note that as a consequence of the uniqueness of the standard bilinear (Cartan-Killing) form on $E_{10}$ there is a unique $E_{10}$ extension of the billiard Hamiltonian (4) given by

$$
\mathcal{H} \rightarrow \mathcal{H}+\sum_{\alpha \in \Delta_{+}\left(E_{10}\right)} \sum_{s=1}^{\operatorname{mult}(\alpha)} e^{-2 \alpha(\beta)} \Pi_{\alpha, s}^{2} .
$$

where the first sum runs over the positive roots $\alpha$ of $E_{10}$. This extended system requires additional constraints. A first step in this direction was taken in [36] where a correspondence was established at low $E_{10}$ levels between the classical canonical constraints of $D=11$ supergravity (in particular, the diffeomorphism and Gauss constraints) on the one hand, and a set of constraints that can be consistently imposed on the $E_{10} / K\left(E_{10}\right)$ coset space dynamics on the other. The fact that the latter can be cast in a 'Sugawara-like' form as quadratic expressions in terms of the $E_{10}$ Noether charges [36] would make them particularly amenable for the implementation on a quantum wavefunction. In addition, one would expect that $P S L_{2}(\mathrm{O})$ must be replaced by a much larger 'modular group' whose action extends beyond the Cartan subalgebra degrees of freedom all the way into $E_{10}$, perhaps along the lines suggested in 37.

As noted above, the inequality (11) implies that $\Phi(\rho, z) \rightarrow 0$ for $\rho \rightarrow \infty$, and hence the wavefunction $\Psi$ vanishes at the singularity, in such a way that the norm is preserved. Its oscillatory nature entails that it cannot be analytically extended beyond the singularity, a result whose implications for the question of singular- 
ity resolution in quantum cosmology remain to be explored. The mechanism usually invoked to resolve singularities in canonical approaches to quantum geometrodynamics would be to replace the classical 'trajectory' in the moduli space of 3-geometries (that is, WDW superspace) by a quantum mechanical wave functional which 'smears' over the singular 3-geometries. By contrast, the present work suggests a very different picture, namely the 'resolution' of the singularity via the effective disappearance (de-emergence) of space-time near the singularity (see also 35] ). The singularity would thus become effectively 'unreachable'. This behavior is very different from other possible mechanisms, such as the Hartle-Hawking no boundary proposal [38], or cosmic bounce scenarios of the type considered recently in the context of minisuperspace loop quantum cosmology [39, 40, 41], both of which require continuing the cosmic wavepacket into and beyond the singularity at $\rho=\infty$.

A key question for singularity resolution concerns the role of observables, and their behavior near the singularity. While no observables (in the sense of Dirac) are known for canonical gravity, we here only remark that for the $E_{10} / K\left(E_{10}\right)$ coset model the conserved $E_{10}$ Noether charges do constitute an infinite set of observables, as these charges can be shown to commute with the full $E_{10}$ Hamiltonian (20). The expectation values of these charges are thus the only quantities that remain welldefined and can be sensibly computed in the deep quantum regime, where the $E_{10} / K\left(E_{10}\right)$ coset model is expected to replace space-time based quantum field theory.

\section{Acknowledgments}

We thank T. Damour, C. Hillmann, A. Rendall and H. Then for discussions. AK is a Research Associate of the Fonds de la Recherche Scientifique-FNRS, Belgium.
[1] V. A. Belinskii, I. M. Khalatnikov and E. M. Lifshitz, Adv. Phys. 19 (1970) 525; Adv. Phys. 31 (1982) 639.

[2] M.P. Ryan and L.C. Shepley, Homogeneous Relativistic Cosmologies, (Princeton University Press, 1975).

[3] C.W. Misner, in Deterministic Chaos in General Relativity, edited by D. Hobill et al. (Plenum Press, New York, 1994).

[4] T. Damour, M. Henneaux and H. Nicolai, Class. Quant. Grav. 20 (2003) R145 arXiv:hep-th/0212256.

[5] M. Henneaux, D. Persson and P. Spindel, Living Rev. Rel. 11 (2008) 1 arXiv:0710.1818 [hep-th]].

[6] T. Damour and M. Henneaux, Phys. Rev. Lett. 86 (2001) 4749 arXiv:hep-th/0012172.

[7] B. S. DeWitt, Phys. Rev. 160 (1967) 1113.

[8] C. W. Misner, Phys. Rev. Lett. 22 (1969) 1071.

[9] C. W. Misner, In: J R Klauder, Magic Without Magic, (W.H. Freeman \& Co, San Francisco, 1972) 441-473.

[10] A. Macias, O. Obregon and M. P. Ryan, Class. Quant. Grav. 4 (1987) 1477.

[11] R. Graham and P. Szepfalusy, Phys. Rev. D42 (1990) 2483.

[12] P. V. Moniz, Int. J. Mod. Phys. A 11 (1996) 4321 arXiv:gr-qc/9604025.

[13] P. D. D'Eath, Supersymmetric quantum cosmology, (Cambridge University Press, Cambridge, U.K., 1996).

[14] A. Csordas and R. Graham, Phys. Rev. Lett. 74 (1995) 4129 arXiv:gr-qc/9502004.

[15] C. Kiefer, Quantum Gravity, (Clarendon Press, Oxford, 2004).

[16] L. A. Forte, Class. Quant. Grav. 26 (2009) 045001 arXiv:0812.4382 [gr-qc]].

[17] T. Damour, M. Henneaux and H. Nicolai, Phys. Rev. Lett. 89 (2002) 221601 arXiv:hep-th/0207267.

[18] We here keep the dimension $d+1$ of space-time arbitrary but will specialize to $D=11$ supergravity below.

[19] Uniqueness properties of the DeWitt metric are discussed in D. Giulini and C. Kiefer, Phys. Lett. A193 (1994) 21.

[20] Note the absence of ordering ambiguities in this expression and the quantized supersymmetry constraint (16).

[21] In contrast to [11, 16], where Neumann boundary condi- tions are assumed.

[22] H. Iwaniec, Spectral methods of automorphic forms, Am. Math. Soc. Graduate Studies in Mathematics Vol. 53 (2002).

[23] A. J. Feingold, A. Kleinschmidt and H. Nicolai, J. Algebra 322 (2009) 1295 arXiv:0805.3018 [math.RT]].

[24] J.H. Conway and D.A. Smith, On quaternions and octonions, (A.K. Peters, Wellesley, MA, 2003).

[25] M. Eie and A. Krieg, Math. Z. 210 (1992) 113-128.

[26] G. Steil, DESY Preprint (1994) DESY 94-028.

[27] D. A. Hejhal, In: D. A. Hejhal et al. (eds.), Emerging applications of number theory, IMA Series No. 109 Springer (1999) 291-315.

[28] For a review of quantum and arithmetical chaos see: E. Bogomolny, nlin/0312061

[29] H. Then, Math. Comp. $74 \quad$ (2004) 363 math-ph/0305047.

[30] R. Aurich, F. Steiner and H. Then, arXiv:gr-qc/0404020

[31] T. Damour, A. Kleinschmidt and H. Nicolai, Phys. Lett. B 634 (2006) 319 arXiv:hep-th/0512163.

[32] S. de Buyl, M. Henneaux and L. Paulot, JHEP 0602 (2006) 056 arXiv:hep-th/0512292.

[33] T. Damour, A. Kleinschmidt and H. Nicolai, JHEP 0608 (2006) 046 arXiv:hep-th/0606105.

[34] T. Damour and C. Hillmann, arXiv:0906.3116 [hep-th].

[35] T. Damour and H. Nicolai, Int. J. Mod. Phys. D 17 (2008) 525 arXiv:0705.2643 hep-th]].

[36] T. Damour, A. Kleinschmidt and H. Nicolai, Class. Quant. Grav. 24 (2007) 6097 arXiv:0709.2691 [hep-th]].

[37] O. Ganor, arXiv:hep-th/9903110

[38] J. B. Hartle and S. W. Hawking, Phys. Rev. D 28 (1983) 2960.

[39] M. Bojowald, Phys. Rev. Lett. 86 (2001) 5227 arXiv:gr-qc/0102069.

[40] A. Ashtekar, A. Corichi and P. Singh, Phys. Rev. D 77 (2008) 024046 arXiv:0710.3565 [gr-qc]].

[41] M. Bojowald, Phys. Rev. Lett. 100 (2008) 221301 arXiv:0805.1192 [gr-qc]]. 